\title{
Emergence and spread of a new community-genotype methicillin-resistant Staphylococcus aureus clone in Colombia
}

\author{
Javier Escobar-Perez ${ }^{1 *}$, Niradiz Reyes ${ }^{2}$, Ricaurte Alejandro Marquez-Ortiz ${ }^{1}$, Juan Rebollo², Hernando Pinzón², \\ Catalina Tovar ${ }^{3}$, Jaime Moreno-Castañeda ${ }^{4}$, Zayda Lorena Corredor ${ }^{1}$, Betsy Esperanza Castro ${ }^{1}$, \\ Maria Victoria Moncada ${ }^{1}$ and Natasha Vanegas ${ }^{1,5}$
}

\begin{abstract}
Background: Community-genotype methicillin-resistant Staphylococcus aureus (CG-MRSA) clones are a global concern due to their resistance and increased virulence and their ability to cause infections both hospitalized patients and healthy people in the community. Here, we characterize 32 isolates of a new CG-MRSA clone. These isolates were identified in four cities in Colombia, South America.

Methods: The isolates were recovered from four different epidemiological and prospective studies that were conducted in several regions of Colombia. Molecular characterizations included multilocus sequence typing; pulsed-field gel electrophoresis; SCCmec, agr and spa typing; and whole-genome sequencing.

Results: All isolates belonged to ST923 (clonal complex 8), harbouring SCCmec IVa and a spa type t1635 and lacking an arginine catabolism mobile element. The isolates were classified as COL923, were resistant to at least one non-betalactam antibiotic, and exhibited high frequencies $(>60 \%)$ of resistance to macrolides and tetracycline. Using wholegenome sequencing, we found that this new clone harbours novel prophage 3 and beta-island structures and a slightly different pathogenicity island 5. Moreover, isolates belonging to the COL923 clone are grouped in a different clade than USA300 and USA300-LV.
\end{abstract}

Conclusion: Our results show the emergence and spread of the COL923 clone in different cities in Colombia. This clone is resistant to several antibiotics and possesses new structures in its mobile genetic elements.

Keywords: Methicillin-resistant Staphylococcus aureus, Community, New clone, Colombia

\section{Background}

The emergence, dissemination and establishment of community-genotype methicillin-resistant Staphylococcus aureus (CG-MRSA) clones is a global concern due to their increased virulence and their enhanced ability to cause infections in healthy people compared with hospitalgenotype MRSA (HG-MRSA) clones. In addition, the frequency of CG-MRSA clones has increased among MRSA infections in hospitalized patients in several countries worldwide [1-4], which implies the replacement of traditional HG-MRSA clones by CG-MRSA clones in the

\footnotetext{
* Correspondence: javiesco21@yahoo.com; escobarjavier@unbosque.edu.co 'Bacterial Molecular Genetics Laboratory, Universidad El Bosque, Carrera 9 \#131A-02, 110121274 Bogotá, DC, Colombia

Full list of author information is available at the end of the article
}

hospital setting and also demonstrates acquisition of resistance to non-beta-lactam antibiotics. USA300 is a CG-MRSA pandemic clone that is circulating in North America [1] but has also been reported on every other continent, including in countries such as Denmark, Spain, France, Italy, Norway, Austria, the United Kingdom, Germany, Japan, Korea, Singapore and Australia [5-8]. This distribution of this clone demonstrates its ability to spread. Since 2004, a CG-MRSA clone that is genetically related to USA300 has been found to cause infections in adults and children in Colombia and several other South American countries $[6,9,10]$. In contrast to USA300, this variant harbours SCCmec IVc (3.1.2) element but lacks an arginine catabolism mobile element (ACME). In recent years, the frequency of this MRSA-IVc clone has increased 
among MRSA infections both in the community and in hospitalized patients, replacing traditional HG-MRSA clones (e.g., Chilean and Brazilian clones) $[11,12]$. We recently reported the detection of eight isolates belonging to a new CG-MRSA clone that have caused paediatric infections in Bogota, Colombia [13]. These isolates were found to possess SCCmec IVa and a spa type t1635; to belonged to the ST923 (a single-locus variant of ST8); to possess an SmaI-restriction pulsed-field gel electrophoresis (PFGE) pulsotype that is not related to that of the USA300 clone ( $>6$ bands of difference); and to lack an ACME, which is frequently found in the USA300 clone. To determinate whether this new clone is circulating in other areas of Colombia, we conducted an active search for this clone and performed a genomic characterization. In this study, we show that this new CG-MRSA clone (named COL923 by our group) circulates in at least four regions of Colombia and possesses new clone-specific molecular characteristics.

\section{Methods}

\section{Bacterial isolate collection and identification}

A total of 32 CG-MRSA isolates (containing SCCmec type IV and/or possessing the gene $l u k S / F-P V$, seq, sek or bsaB) belonging to the new clone COL923 (SCCmec IVa, spa type t1635 and ST923) were identified in four different studies (Additional file 1: Table S1). In the first study, $430 \mathrm{~S}$. aureus isolates were prospectively and systematically recovered from infections in paediatric patients $(<18$ years old) at a hospital in Cartagena, which is a city on the Caribbean coast. Nineteen of the MRSA isolates were related to COL923 (one isolate per patient). In the second study, MRSA nasal colonization was investigated in 2867 healthy adult individuals ( $>18$ years old) living in 10 regions that are geographically distant from Colombia. Of the isolates obtained, three isolates from two different regions were related to COL923. In the third study, MRSA nasal colonization was investigated in 150 healthy children ( $<5$ years old) in Monteria, which is a city in northwest Colombia. Of the isolates obtained, six isolates were related to COL923. Finally, in the fourth study, MRSA nasal colonization was evaluated in 205 healthy children (5 to 14 years old) in Cartagena, and four CG-MRSA isolates were found to belong to the new clone.

\section{Antimicrobial susceptibility testing}

The profile of susceptibility to 12 antibiotics (oxacillin, gentamicin, rifampicin, erythromycin, ciprofloxacin, vancomycin, linezolid, tetracycline, clindamycin, chloramphenicol, trimethoprim and sulfamethoxazole) was determined for each of the isolates using the agar dilution method. The results were interpreted according to the 2015 guidelines of the Clinical and Laboratory
Standards Institute (CLSI). Inducible clindamycin resistance was also determined using the D-test.

\section{Molecular characterization and establishment of genetic relatedness of isolates}

The SCCmec type and subtype were established for each clone using multiplex PCR, as previously described [14]. The lukS/F-PV, etb, eta, hlg, sea, seb, sec, seg, seh, sei, sej, sek, sel, sem, sen, seo, sep and seq genes were also analysed using PCR [15]. In addition, the presence of the blaZ, tetK, tetM, ermA, ermB, ermC, msrA, mph and $m e f A$ genes was evaluated in all isolates. Genetic relatedness between the isolates was determined by PFGE. The obtained pulsotypes were interpreted according to the percentage of similarity and the criteria proposed by Tenover and colleagues [16]. The agr group, spa type and multilocus sequence typing were determined as previously reported [17].

\section{Detection of mobile genetic elements (MGEs) and genome sequencing of new CG-MRSA clone}

The presence or absence of the most important MGEs and genomic islands (GIs) was evaluated using different PCR strategies (Additional file 2: Figure S1). For the genome sequencing analysis, isolates were selected as follows: for the COL923 clone, two representative isolates were selected for each of the two main PFGE pulsotypes (isolates 5sau489 and 17sau368, belonging to the first PFGE pulsotype (Fig. 1), and isolates 5 sau410 and 17sau58, belonging to the second PFGE pulsotype (Fig. 1)). In addition, Col131 was selected because it was the first isolate related to the COL923 clone that was identified in Colombia. For the USA300-LV clone (the most frequent CG-MRSA clone in Colombia), one representative isolate was selected for each of the four most frequent PFGE pulsotypes (i.e., 17sau599, 5sau003/ 17sau366, 17sau193 and 17sau391). Total DNA was extracted from the 10 MRSA clinical isolates using the PureLink ${ }^{\bullet}$ Genomic DNA Mini Kit from Thermo Fisher. The DNA was used to prepare multiplexed total DNA libraries using the Nextera XT Dual Index Sequencing Primer Kit (Illumina, Inc.) and the KAPA Library Amplification Kit (Kapa Biosystems). The multiplexed libraries were pooled and sequenced by paired-end sequencing using the Illumina MiSeq (2x300 cycles) and HiSeq (2x101 cycles) platforms. The libraries were assembled using SOAPdenovo2 V2.04-r240, with kmer values of 63 and 127 for HiSeq and MiSeq, respectively. The basic assembly statistics are shown in Additional file 3: Table S2. The reads were also mapped to the USA300-FPR3757 genome using SHRiMP 2.2.3 and were then compared and visualized using BRIG software $[18,19]$. The genetic relatedness of the sequenced isolates was established using phylogenetic analysis. To build a phylogenetic 


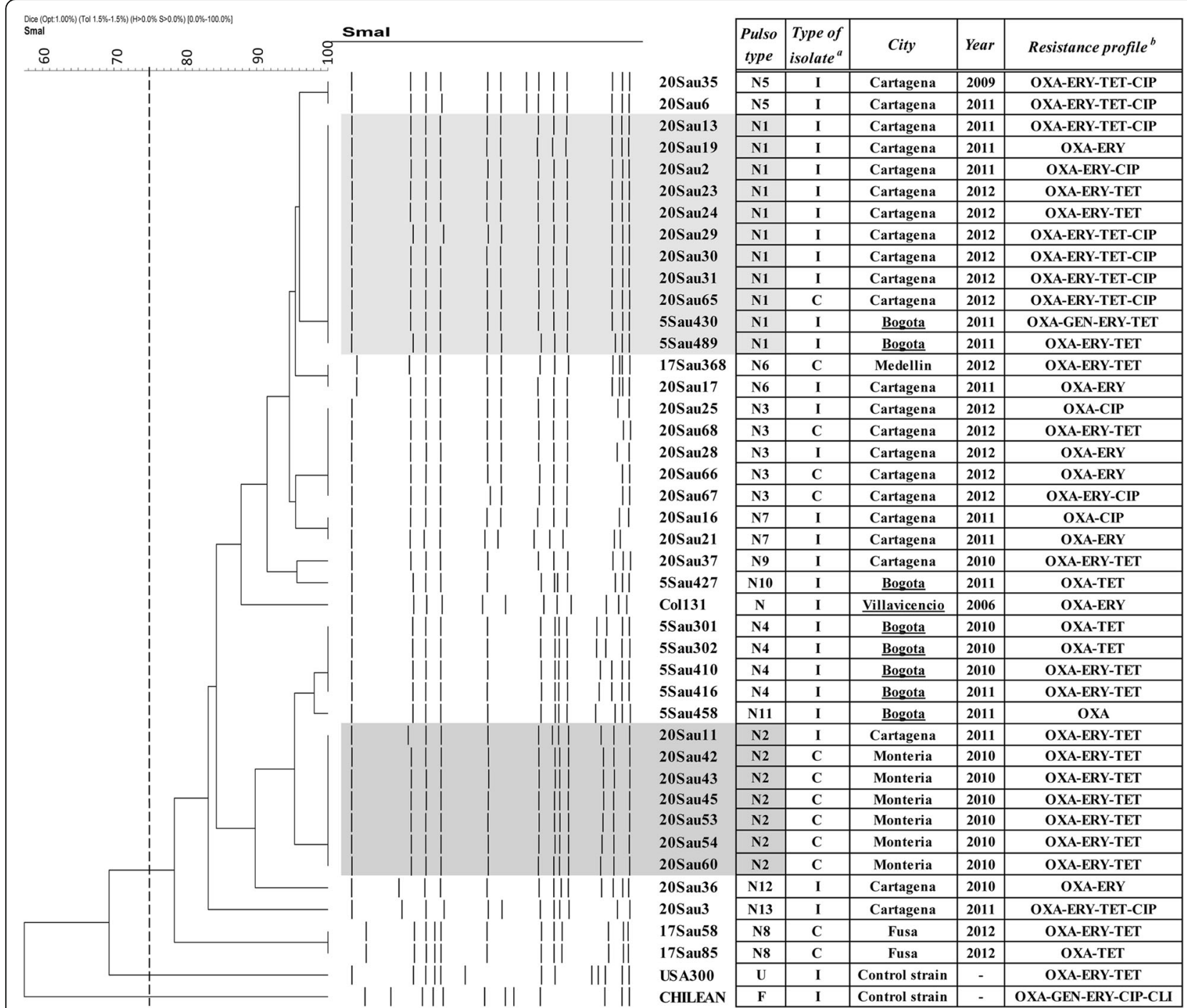

Fig. 1 Genetic relatedness among isolates belonged to the COL923 clone identified in Colombia. The isolates underlined correspond to the isolates identified previously. USA300-0114-FPR3757 and CHL93 (Chilean clone) strains were used as standards. PFGE patterns were considered different when they showed a similarity lower than $75 \%$ (broken line). Shaded region indicates the two more frequent pulsotypes. $a$. Type of isolate: I = infection, C = Colonization. b. OXA = oxacillin, TET = tetracycline, ERY = erythromycin, GEN = gentamicin, CIP = ciprofloxacin, CLI =clindamycin

tree, the partially assembled (i.e., 17sau193, 17sau366, 17sau368, 17sau391, 17sau58, 17sau599, 5sau003, 5sau410, 5sau489 and Col131) and reference (i.e., USA300 and NCTC8325) genomes were annotated using Prokka [20] and an alignment was created for 2288 concatenated core genes (genes with $\geq 99 \%$ nucleotide identity that were present in all genomes) using Roary [21] and PRANK [22]. Poorly aligned positions and divergent regions were eliminated using Gblocks [23]. Finally, the phylogenetic tree was created using RAxML version 8.2.9 [24] by running 1000 bootstrap replicates under the generalized timereversible model (GTRCAT). Finally, a consensus tree was plotted using Dendroscope [25]. Branch lengths are expressed in units of changes/nucleotide position (scale bar). The NCTC8325 strain was included as an outgroup control (GenBank ID: NC_007795.1). Different MGEs were identified from the mapping analysis and then annotated using the RAST server [26].

\section{Results}

Among the 32 CG-MRSA isolates belonging to the COL923 clone that were analysed, 19 were recovered from paediatric infections, and 13 were recovered from nasal samples from healthy youths and children. Among the 19 infection isolates, one was identified in 2009; two, in 2010; nine, in 2011; and seven, in 2012. The main clinical diagnosis was skin or soft-tissue infection (10 cases). Four patients developed septic shock, and of these, one also developed pneumonia (Table 1). Eighteen 
Table 1 Demographic and clinical characteristics of paediatric patients with MRSA infections

\begin{tabular}{ll}
\hline $\begin{array}{l}\text { Demographic data and clinical } \\
\text { characteristics }\end{array}$ & $\begin{array}{l}\text { All subjects }(n=19) \\
\mathrm{n}(\%)\end{array}$ \\
\hline Age (years) (range) & $8.3(0.17-14)$ \\
Age distribution & \\
$\quad$ Newborn & $0(0.0)$ \\
Infants & $1(5.3)$ \\
Pre-school age & $4(21.1)$ \\
School age & $7(36.8)$ \\
Adolescents & $7(36.8)$ \\
Male sex, \% & $12(63.0)$
\end{tabular}

$\begin{array}{ll}\text { Year of recovery (time in months) } & \\ 2009(3) & 1(5.3) \\ 2010(12) & 2(10.5) \\ 2011(12) & 9(47.4) \\ 2012(6) & 7(36.8) \\ \text { Admission Site } & \end{array}$

Emergency room $19(100.0)$

Clinical diagnosis

$\begin{array}{ll}\text { SSTI }^{\mathrm{b}} & 10(43.8) \\ \text { Osteoarticular infection } & 5(26.0) \\ \text { Pneumonia } & 1(5.6) \\ \text { Septic Shock } & 3(2.5) \\ \text { Onset time of symptoms (mean, SDC), days } & 17.4(20.0) \\ \text { Hospital management }^{\mathrm{d}} & \\ \text { Hospitalized- drainage } & 15(69.1) \\ \text { PICU } & 4(24.1)\end{array}$

Empirical antimicrobial therapy ${ }^{\mathrm{e}}$

$\begin{array}{ll}\text { CLI } & 13(68.4) \\ \text { OXA } & 2(10.5) \\ \text { CLI/RIF } & 2(10.5) \\ \text { RIF } & 1(5.3) \\ \text { CLI/RIFNAN } & 1(5.3) \\ \text { Definitive antimicrobial therapye } & \\ \text { SXT } & 11(57.9) \\ \text { CLI/RIF } & 3(15.8) \\ \text { OXA } & 1(5.3) \\ \text { RIF } & 1(5.3) \\ \text { CLI } & 1(5.3) \\ \text { CLI/RIFNAN } & 1(5.3) \\ \text { VAN/LZD/SXT } & 1(5.3)\end{array}$

Clinical outcome

$$
\text { Improvement } 19 \text { (100.0) }
$$

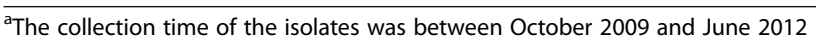
${ }^{\mathrm{b}} \mathrm{SSTI}$ Skin and soft tissue infection

'SD standard deviation

${ }^{\mathrm{d}}$ PICU paediatric intensive care unit

${ }^{\text {e}} \mathrm{CLI}$ clindamycin, OXA oxacillin, RIF rifampicin, SXT trimethoprim-sulfamethoxazole, $V A N$ vancomycin, $L Z D$ linezolid patients were definitively treated for MRSA infections with active antimicrobials, and one was treated with oxacillin. Thirteen patients (68.4\%) were additionally managed with incision and drainage with full recovery observed in all 13 cases. Four patients $(21.05 \%)$ required a paediatric intensive care unit stay because of the severity of their infections. All (100\%) infections were identified within the first $48 \mathrm{~h}$ of hospital admission in children without healthcare-associated risk factors who entered the hospital through the emergency department (i.e., community-onset MRSA infections).

In contrast, the 13 MRSA nasal colonization isolates were identified and recovered from healthy people living in Medellin, Cartagena, Monteria and Fusagasuga, which are four cities both distant from each other and distant from Bogota and Villavicencio, where the COL923 strain was first reported. The fact that we did not find any epidemiological link among the people harbouring this clone suggested that this CG-MRSA clone was already circulating in several regions of the country.

\section{Antimicrobial resistance profiles and resistance mechanisms}

All isolates were susceptible to vancomycin, linezolid, clindamycin, gentamicin, rifampicin, chloramphenicol and trimethoprim/sulfamethoxazole. However, the minimal inhibitory concentration (MIC) for vancomycin was $1 \mathrm{mg} /$ L. Of the 32 COL923 isolates, 30 (93.8\%), 21 (65.6\%) and $12(37.5 \%)$ were resistant to erythromycin, tetracycline and ciprofloxacin, respectively (Table 2). Additionally, all isolates were resistant to at least one non-beta-lactam antibiotic. The most frequent multiresistance profiles consisted of erythromycin-tetracycline resistance $(40.6 \%)$ or erythromycin-tetracycline-ciprofloxacin resistance (25.0\%) (Table 2). The blaZ gene was detected in all of the isolates. All erythromycin-resistant isolates (30) presented an M phenotype and harboured the $m s r A$ and $m p h C$ genes. The 21 isolates that were resistant to tetracycline harboured the tet $K$ gene. The blaZ, tetK, $m s r A$ and $m p h C$ genes were localized in contigs that did not map to the USA300FPR3757 genome, suggesting the possibility of transmission by plasmids, although this needs to be confirmed in future work.

\section{Molecular characteristics and genetic relatedness of isolates}

The molecular characterization of the 32 CG-MRSA isolates demonstrated that they all harboured SCCmec IVa (IV.1.1.1), were of spa type t1635 (YHGFMBO) and presented a 54-bp insertion in the sausa300_0808 gene in $S$. aureus pathogenicity island 5 (SaPI5). These same features were previously found in the COL923 isolates recovered from paediatric infections in Bogota, Colombia [13]. All isolates harboured the sek and seq genes within 
SaPI5. The $l u k S / F-P V$ genes were detected in 29 (90.6\%) isolates. An ACME was not detected in any of the isolates. The PFGE analysis of the 32 isolates revealed the presence of 10 different pulsotypes (the N1 to N3, N5 to N9, N12 and N13 pulsotypes, as shown in Fig. 1). The $\mathrm{N} 1$ and $\mathrm{N} 2$ pulsotypes were the most frequent, found in 9 (28.1\%) and 7 (21.9\%) isolates, respectively (Table 2 ). All 32 isolates included in this study showed similarities greater than $80 \%$ with respect to the isolates previously recovered in Bogota [12] (Fig. 1). Finally, all isolates belonged to agr group I and the sequence type 923 .

\section{Detection of MGEs, Gls and genome comparison}

The assembly statistics for the sequenced genomes are shown in Additional file 3: Table S2. The genomic mapping of the COL923 clone revealed certain differences with respect to the USA300-FPR3757 and USA300-LV clones (Fig. 2). These differences were mainly due to changes in the MGEs (e.g., changes in the prophage 3, beta-island, and SCCmec J3 regions and the absence of ISsau5) in addition to single-nucleotide polymorphisms in the core genome. It is also important to highlight that the beta-island $(v \mathrm{Sa} \beta)$ was smaller in the COL923 clone $(13,980 \mathrm{bp})$ than in the USA300-FPR3757 and USA300LV (37,590 bp) clones, displaying a truncated structure. PCR analysis showed that all isolates (except Col131) possessed this truncated beta-island $(v \mathrm{Sa} \beta)$ structure
(Fig. 2a and Fig. 3b and c). All isolates harboured the sak gene (a marker of prophage 3), and genome sequencing confirmed the presence of prophage 3 ( $\phi S A 3)$ but indicated that it possessed a new genetic structure that best matched the NCTC8325 strain (63.4\% identity) (Fig. 3a). However, this prophage 3 ( $\phi S A 3)$ shared low sequence identity with the USA300-FPR3757 (38.4\%) and N315 (39.6\%) prophage 3 ( $\phi \mathrm{SA} 3)$. Additionally, the genome sequencing analysis of 5 sau 489 revealed two DNA insertions in the SCCmec J3 region that were not present in USA300-FPR3757 (Fig. 3d). The first insertion (578 bp) harboured an open reading frame (ORF) that encoded a putative transposase, which had previously been reported in another CG-MRSA-ST72-IVa strain that was identified in South Korea [27]. PCR analysis showed that all isolates (including the 32 isolates described in this study and 9 isolates identified previously) contained two DNA insertions in the $\mathrm{J} 3$ region of SCCmec (Fig. 3e). These findings show that the COL923 clone possesses SCCmec IVa that is not identical to that in USA300.

The genetic structure of $v \mathrm{Sa} \alpha$ in the isolates described in this study was the same as that reported for the USA300 clone [28]. A maximum-likelihood phylogenetic analysis of the core genomes showed that the isolates could be segregated into two distinct clades (Fig. 2c). Clade 1 consisted of isolates belonging to the USA300LV clone (ST8-IVc), while clade 2 consisted of isolates

Table 2 Antimicrobial resistance and molecular characteristics of MRSA isolates belonging to the COL923 clone

\begin{tabular}{|c|c|c|c|}
\hline \multirow{2}{*}{$\begin{array}{l}\text { Characteristics of } \\
\text { isolates }\end{array}$} & All isolates $(n=32)$ & Infection $(n=19)$ & Colonization $(n=13)$ \\
\hline & n (\%) & n (\%) & n (\%) \\
\hline \multicolumn{4}{|c|}{ Antimicrobial resistance ${ }^{a}$} \\
\hline ERY & $30(93.8)$ & $17(89.5)$ & $13(100.0)$ \\
\hline TET & $21(65.6)$ & $11(57.9)$ & $10(76.9)$ \\
\hline CIP & $12(37.5)$ & $10(52.6)$ & $2(15.4)$ \\
\hline \multicolumn{4}{|c|}{ Multiple antibiotics resistance profiles } \\
\hline OXA-ERY-TET & $13(40.6)$ & $4(21.1)$ & $9(69.2)$ \\
\hline OXA-ERY-TET-CIP & $8(25.0)$ & $7(36.8)$ & $1(7.7)$ \\
\hline OXA-ERY & $7(21.9)$ & $5(26.3)$ & $2(15.4)$ \\
\hline OXA-CIP & $2(6.3)$ & $2(10.5)$ & $0(0.0)$ \\
\hline OXA-ERY-CIP & $2(6.3)$ & $1(5.3)$ & $1(7.7)$ \\
\hline Only OXA & $0(0.0)$ & $0(0.0)$ & $0(0.0)$ \\
\hline \multicolumn{4}{|c|}{ Main PFGE pulsotype ${ }^{c}$} \\
\hline N1 & $9(28.1)$ & $8(42.1)$ & $1(7.7)$ \\
\hline N2 & $7(21.9)$ & $1(5.3)$ & $6(46.2)$ \\
\hline N3 & $5(15.6)$ & $2(10.5)$ & $3(23.1)$ \\
\hline N5 & $2(6.3)$ & $2(10.5)$ & $0(0.0)$ \\
\hline N6 & $2(6.3)$ & $1(5.3)$ & $1(7.7)$ \\
\hline
\end{tabular}

aOX oxacillin, TET tetracycline, ERY erythromycin, GEN gentamicin, CIP ciprofloxacin, CLI clindamycin

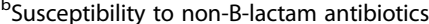

${ }^{\mathrm{C}} \mathrm{N} 4$ pulsotype was assigned to isolates recovered from Bogota (Fig. 1) 


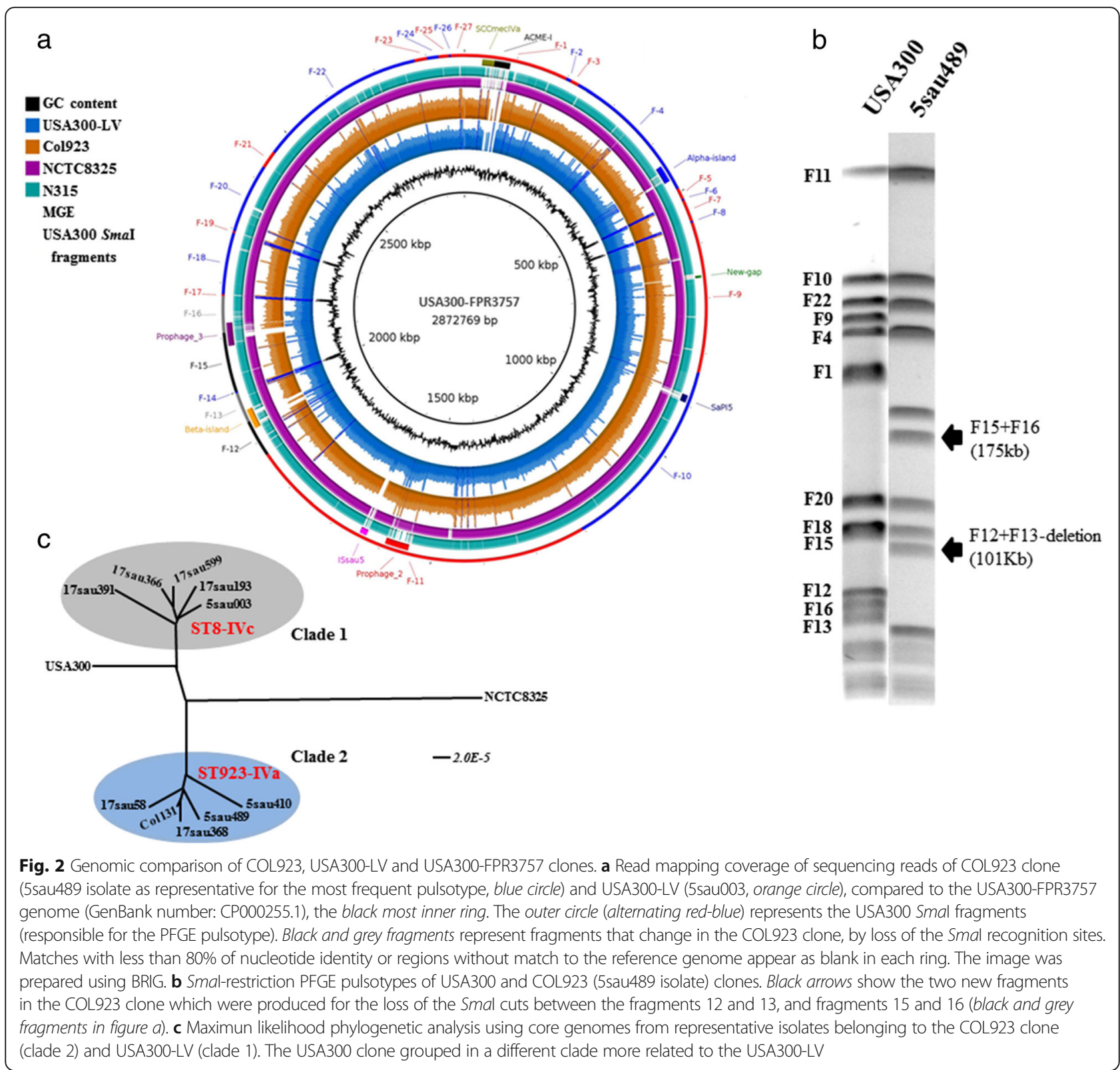

belonging to the COL923 clone (ST923-IVa). The USA300 clone was not part of any clade; nevertheless, it was more closely related to the isolates in the USA300LV clade. Taken together, these data strengthen the idea that the COL923 clone is genetically different with respect to the USA300 and USA300-LV clones, in addition to the differences observed in MGEs.

\section{Discussion}

The extraordinary genetic success of CG-MRSA clones is now well known, and we have observed that its frequency has increased during the last two decades, first in the community and then in the hospital setting, in several countries around the world. In Colombia, several genetic and molecular analyses have shown that the most prevalent CG-MRSA clone (USA300-LV) possesses certain characteristics that are similar to those of the USA300 pandemic clone. However, the Colombian CGMRSA clone harbours a different SCCmec (IVc or IV.3.1.2) and, interestingly, does not possess an ACME [6, 11, 29-31]. Between 2008 and 2011, the prevalence of this clone was reported to comprise $70 \%$ and $90 \%$ of MRSA infections in adults and paediatric patients, respectively $[6,11,30]$, in several Colombian cities. However, in 2010, we identified eight CG-MRSA isolates with different molecular and genetic characteristics compared to the USA300-LV clone but with similarity to the COL923 clone [12, 13]. Our data showed that the 


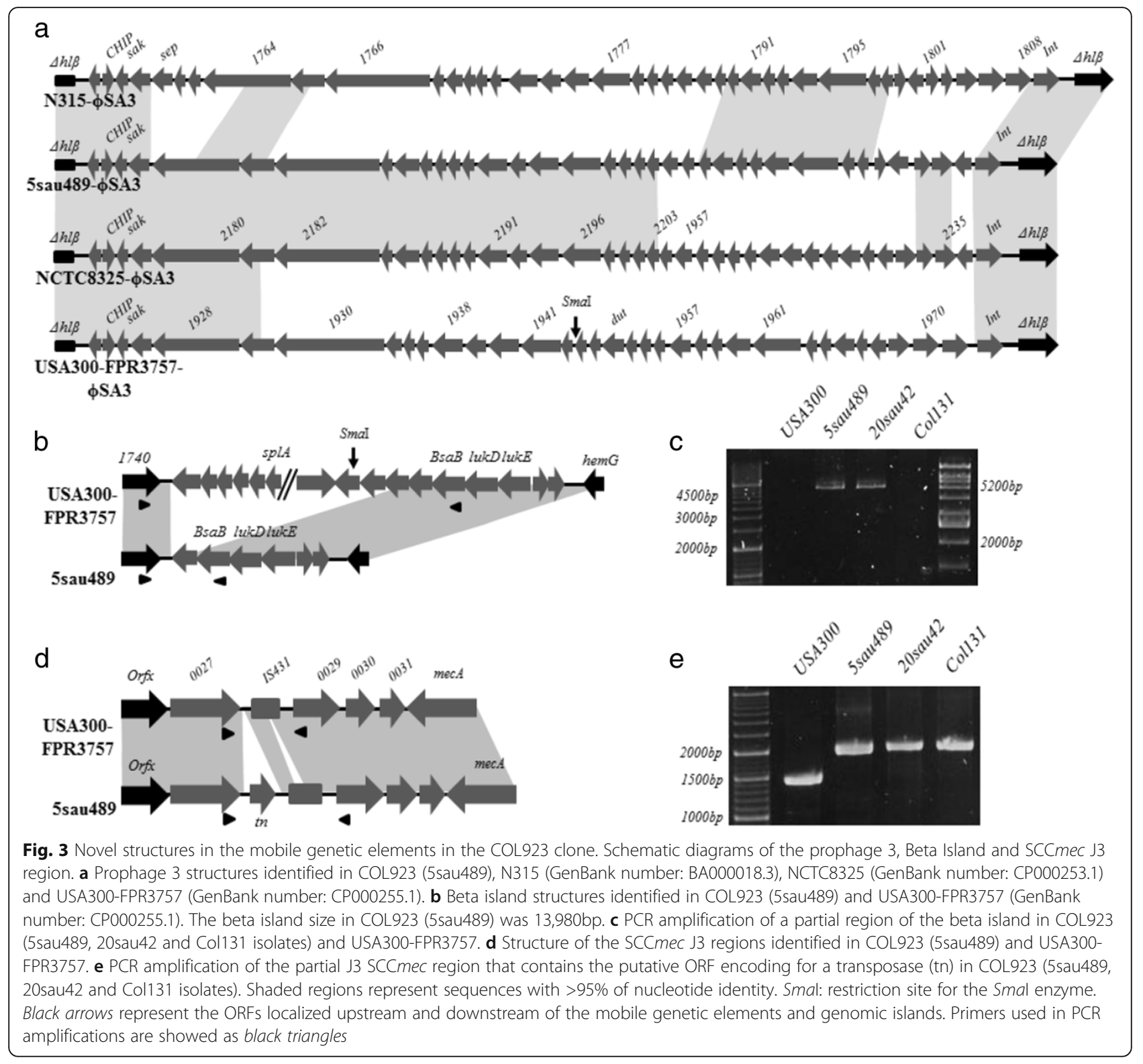

COL923 (CG-MRSA-IVa-ST923) clone had also been causing infections in children in other regions of Colombia (outside Bogota) since 2009. This new clone was identified in five distant geographic areas, which suggests that it was already circulating in several regions of our country and that its emergence represents a dissemination event, and not an epidemic event in one city [11, 29, 32].

In addition, whole-genome sequencing analysis revealed unique characteristics in both the MGE and the core genome of this new CG-MRSA clone. With respect to SCCmec, certain variations were found in its J3 region (Figs. 2 and 3d). For example, a 578-bp insertion was identified that has also been recently reported in SCCmec IVa element harboured in an ST72 (CC8, triple-locus variant of ST8) clinical isolate that caused a community-onset infection [27]. This particular region contains an ORF that encodes a putative transposase. Although the SCCmec IVa element in the COL923 clone still has high nucleotide identity (>98\%) with the SCCmec IVa element in the USA300 clone, our results show that it has also acquired certain foreign DNA fragments $[12,13]$. These results reinforce the idea that the new variant (COL923) has gained DNA fragments and support the importance of further studies aimed at increasing our understanding of this process.

The novel results of the present study include the identification of new prophage 3 (\$SA3) and beta-island $(v \mathrm{Sa} \beta)$ structures in the COL923 clone. To our knowledge, these structures have not been previously 
reported. According to these results, the new prophage 3 has low genetic relatedness to the prophage 3 that was previously identified in the USA300 clone. Based on its structure and size, this new prophage can be classified into the Siphoviridae family, and it combines parts of other prophages into a mosaic structure. Thus, we could hypothesize that it originated during a series of events that involved recombination among the functional modules of different prophage 3 types, a process that has been previously reported in Siphoviridae prophages [33]. The insertion of this new prophage 3 and a truncated beta-island caused the loss of two SmaI restriction sites in the chromosome of the new clone, leading to a change in the PFGE pulsotype (Fig. 2).

In 2006, Alvarez et al. reported recovering CG-MRSA isolates (Col131) from an infection in an adult patient in 2004 [9, 34], Subsequent molecular and genetic analyses showed that this Col131 isolate harboured SCCmec IVa. The molecular analysis of the Col131 isolate that we performed showed that it shared molecular characteristics with the new COL923 clone (ST923, spa type t1635, sausa300_0808-variant gene, prophage 3, SaPI5 and J3SCCmec structure) but had a different PFGE pulsotype (Fig. 1). Interestingly, since 2004, the USA300-LV clone has shown increasing frequency in MRSA infections, whereas the COL923 clone has shown a very low frequency. These findings suggest two possible hypotheses. First, the Col131 isolate, after acquiring SCCmec IVa, may have undergone several genetic changes that resulted in environmental advantages that gave rise to the COL923 isolates identified since 2009. Second, certain ST923 methicillin-sensitive S. aureus isolates with similar molecular characteristics and genetic relatedness to the Col131 isolate may have acquired the same SCCmec IVa during different events.

It is important to highlight that among the COL923 isolates identified here, four were resistant to the non-betalactam antibiotics ciprofloxacin, erythromycin and tetracycline. The last two types of resistance are associated with the $m s r A, m p h C$ and tet $K$ genes, which are possibly carried by plasmids (these were not mapped in the reference chromosomes), suggesting that plasmids are mobilizing among CG-MRSA clones present within the community in Colombia. We hypothesize that both selective pressure due to the overuse of antibiotics and the unnatural accumulation of certain substances in the community have filtered the local $S$. aureus population to favour the new variant, which likely has better fitness because of its $m s r A$ - or tetKpositive plasmid. In comparison with the USA300 and USA300-LV clones circulating in our region, the COL923 clone displays a broader resistance range and an MIC to vancomycin of $1 \mathrm{mg} / \mathrm{L}$, even outside the hospital setting. The rise in the frequency of this clone is therefore a possible challenge to the health system.

\section{Conclusion}

Our data demonstrate that a new CG-MRSA COL923 clone is circulating in different regions of Colombia, rather than being confined to a single city. Additionally, this clone possesses new genetic structures that have not been previously reported that differentiate it from the USA300-LV and USA300 clones. The COL923 clone is a new CG-MRSA clone that is causing infections in people in the community and that is also having an impact within the health system. It has been frequently observed that although many clones possess the ability to acquire the same SCCmec, only a few prevail, with certain clones predominating in their respective geographic niches [35]. This finding suggests that specific genetic determinants govern the predominance of different clones in different regions of the globe. Thus, it is necessary to continue surveillance studies in both the community and hospitals to assess the clinical, economic and social impacts of the dissemination of this new CG-MRSA clone.

\section{Additional files}

\section{Additional file 1: Table S1.}

Additional file 2: Figure S1. Schematic diagram used to detect the main Mobile Genetic Elements (EMG) in the MRSA isolates. The genome sequence reported to USA300-FPR3757 (GenBank accession number CP000255.1), COL (GenBank accession number CP000046.1), Mu50 (GenBank accession number BA000017.4) and N315 (GenBank accession number BA000018.3) were used as reference. Black arrows represent the ORFs localized upstream and downstream of the EGM and GI. Black triangles represent the PCR primers localization. Abbreviations: SaPI: Staphylococcus aureus Pathogenicity Island, $\phi S A:$ S. aureus Prophage, vSad: S. aureus genomic island Alfa, vSaß: S. aureus genomic island beta and vSa4: S. aureus genomic island 4. (TIF 608 kb) Additional file 3: Table S2. Assembly statistics to $10 \mathrm{MRSA}$ isolates sequenced using the MiSeq platform (except 5 sau003 and 5sau489, sequenced using HiSeq 2000). L50 and L75 are the number of contigs greater than N50 and N70 respectively. (DOCX $15 \mathrm{~kb}$ )

\section{Abbreviations}

ACME: Arginine catabolic mobile element; agr: Accessory gene regulator; CGMRSA: Community-genotype methicillin-resistant Staphylococcus aureus; CLSI: Clinical and Laboratory Standards Institute; Gl: Genomic islands; HGMRSA: Hospital-genotype methicillin-resistant Staphylococcus aureus; MGE: Mobile genetics elements; MLST: Multilocus sequence typing; MSSA: Methicillin-sensible Staphylococcus aureus; ORF: Open reading frame; PCR: Polymerase chain reaction; PFGE: Pulsed-field gel electrophoresis; SaPI5: Staphylococcus aureus pathogenicity island 5; SCCmec: Staphylococcal cassette chromosome mec; spa: Staphylococcal protein A; ST: Sequence type; UPGMA: Unweighted Pair Group Method with Arithmetic mean; USA300LV: USA300-latinAmerican variant; WGS: Whole-genome sequencing

\section{Acknowledgments \\ We specially thank to the Vice Chancellery for Research of the El Bosque University (especially to Dr Miguel Otero for your invaluable support), University of Cartagena and University of Sinu. We also thank to Dr Nancy Mugridge, Senior Scientist of Alliances EnGenelC Ltd, Australia, for your invaluable suggestions to the paper.}

\section{Funding}

This work was financially supported by the Administrative Department of Science, Technology and Innovation/Colciencias grant \# 1107-519-28910 Contract \#255-2010, University of Cartagena grant \#RES4776 - 2010; and partially by Colciencias grant 1308-519-29126. 


\section{Availability of data and materials}

The nucleic acids sequences were deposited at National Center for Biotechnology Information (NCBI): GenBank accession numbers KX232514, KX232515 and KX232516. The raw reads from whole genome sequencing to all genomes included were deposited in SRA database in the Bioproject PRJNA342178 under the accession numbers: SRR4195752, SRR4195753, SRR4195754, SRR4195755, SRR4195756, SRR4195757, SRR4195758, SRR4195759, SRR4195761 and SRR4195761, to the samples: 17sau193, 17sau366, 17sau368, 17sau391, 17sau58, 17sau599, 5sau003, 5sau410, 5sau489, Col131, respectively.

\section{Authors' contributions}

JEP, NR, JMC, CT and NV conceived, design and performed the studies; they analysed the data and approved the manuscript; NR, JR and HP carried out the study in Cartagena city, CT carried out the study in Monteria city; RAMO, $J R, Z L C, B E C$ and MVM participated in field in the different cities and carried out the lab work; all authors contributed to interpretation of data, critical review and approval of the final manuscript.

\section{Competing interests}

The authors declare that they have no competing interests.

\section{Consent for publication}

Not applicable.

\section{Ethics approval and consent to participate}

The study and protocol were approved by the Institutional Ethics Committees of all participant institutions (El Bosque University, University of Cartagena and University of Sinu). The protocol was presented to all participants and written and signed informed consent was obtained, for children $<18$ years of age, informed consent was obtained from parents or guardian. In addition, information and records of all participants included in the study were processed anonymously.

\section{Author details}

'Bacterial Molecular Genetics Laboratory, Universidad El Bosque, Carrera 9 \#131A-02, 110121274 Bogotá, DC, Colombia. ${ }^{2}$ Grupo de Genética y Biología Molecular, Universidad de Cartagena, Cartagena, Colombia. ${ }^{3}$ Grupo de Investigación en Enfermedades Tropicales y Resistencia Bacteriana, Montería, Colombia. ${ }^{4}$ Grupo de Microbiología, Instituto Nacional de Salud, Bogotá, Colombia. ${ }^{5} 13$ Institute, Faculty of Science, University of Technology, Sydney, Australia.

Received: 13 May 2016 Accepted: 6 January 2017

Published online: 31 January 2017

\section{References}

1. Diekema DJ, Richter SS, Heilmann KP, Dohrn CL, Riahi F, Tendolkar S, et al. Continued emergence of USA300 methicillin-resistant Staphylococcus aureus in the United States: results from a nationwide surveillance study. Infect Control Hosp Epidemiol. 2014;35:285-92.

2. Otter JA, French GL. Community-associated meticillin-resistant Staphylococcus aureus strains as a cause of healthcare-associated infection. J Hosp Infect. 2011;79:189-93.

3. Alvarez CA, Yomayusa N, Leal AL, Moreno J, Mendez-Alvarez S, Ibanez M, et al. Nosocomial infections caused by community-associated methicillin-resistant Staphylococcus aureus in Colombia. Am J Infect Control. 2010;38:315-8.

4. Park SH, Park C, Yoo JH, Choi SM, Choi JH, Shin HH, et al. Emergence of community-associated methicillin-resistant Staphylococcus aureus strains as a cause of healthcare-associated bloodstream infections in Korea. Infect Control Hosp Epidemiol. 2009;30:146-55.

5. Otto M. Basis of virulence in community-associated methicillin-resistant Staphylococcus aureus. Annu Rev Microbiol. 2010;64:143-62.

6. Reyes J, Rincon S, Diaz L, Panesso D, Contreras GA, Zurita J, et al. Dissemination of methicillin-resistant Staphylococcus aureus USA300 sequence type 8 lineage in Latin America. Clin Infect Dis. 2009;49:1861-7.

7. Blanco R, Tristan A, Ezpeleta G, Larsen AR, Bes M, Etienne J, et al. Molecular epidemiology of Panton-Valentine leukocidin-positive Staphylococcus aureus in Spain: emergence of the USA300 clone in an autochthonous population. J Clin Microbiol. 2011;49:433-6.
8. Nimmo GR. USA300 abroad: global spread of a virulent strain of community-associated methicillin-resistant Staphylococcus aureus. Clin Microbiol Infect. 2012;18:725-34.

9. Alvarez CA, Barrientes OJ, Leal AL, Contreras GA, Barrero L, Rincon S, et al. Community-associated methicillin-resistant Staphylococcus aureus, Colombia. Emerg Infect Dis. 2006;12:2000-1.

10. Alvarez-Olmos Ml, Enriquez SP, Perez-Roth E, Mendez-Alvarez S, Escobar J, Vanegas $\mathrm{N}$, et al. Pediatric cases from Colombia caused by a Panton-Valentine Leukocidin-positive community-acquired methicillin-resistant Staphylococcus aureus ST8-SCCmecIVc clone. Pediatr Infect Dis J. 2009;28:935.

11. Jimenez JN, Ocampo AM, Vanegas JM, Rodriguez EA, Mediavilla JR, Chen L, et al. CC8 MRSA strains harboring SCCmec type IVC are predominant in Colombian hospitals. PLoS One. 2012;7:e38576.

12. Marquez-Ortiz RA, Alvarez-Olmos MI, Escobar Perez JA, Leal AL, Castro BE, Marino AC, et al. USA300-related methicillin-resistant Staphylococcus aureus clone is the predominant cause of community and hospital MRSA infections in Colombian children. Int J Infect Dis. 2014;25:88-93.

13. Escobar JA, Marquez-Ortiz RA, Alvarez-Olmos MI, Leal AL, Castro BE, Vanegas $\mathrm{N}$, et al. Detection of a new community genotype methicillin-resistant Staphylococcus aureus clone that is unrelated to the USA300 clone and that causes pediatric infections in Colombia. J Clin Microbiol. 2013;51:661-4.

14. Milheirico C, Oliveira DC, de Lencastre H. Multiplex PCR strategy for subtyping the staphylococcal cassette chromosome mec type IV in methicillin-resistant Staphylococcus aureus: 'SCCmec IV multiplex'. J Antimicrob Chemother. 2007;60:42-8.

15. Jarraud S, Mougel C, Thioulouse J, Lina G, Meugnier H, Forey F, et al. Relationships between Staphylococcus aureus genetic background, virulence factors, agr groups (alleles), and human disease. Infect Immun. 2002;70:631-41.

16. Tenover FC, Arbeit RD, Goering RV, Mickelsen PA, Murray BE, Persing DH, et al. Interpreting chromosomal DNA restriction patterns produced by pulsed-field gel electrophoresis: criteria for bacterial strain typing. J Clin Microbiol. 1995;33:2233-9.

17. Enright MC, Day NP, Davies CE, Peacock SJ, Spratt BG. Multilocus sequence typing for characterization of methicillin-resistant and methicillin-susceptible clones of Staphylococcus aureus. J Clin Microbiol. 2000;38:1008-15.

18. David M, Dzamba M, Lister D, llie L, Brudno M. SHRiMP2: sensitive yet practical SHort Read Mapping. Bioinformatics. 2011;27:1011-2.

19. Alikhan NF, Petty NK, Ben Zakour NL, Beatson SA. BLAST Ring Image Generator (BRIG): simple prokaryote genome comparisons. BMC Genomics. 2011;12:402.

20. Seemann T. Prokka: rapid prokaryotic genome annotation. Bioinformatics. 2014;30:2068-9.

21. Page A, Cummins CA, Hunt M, Wong VK, Reuter S, Holden MT, et al. Roary: rapid large-scale prokaryote pan genome analysis. Bioinformatics. 2015;31:3691-3.

22. Loytynoja A. Phylogeny-aware alignment with PRANK. Methods Mol Biol. 2014;1079:155-70.

23. Talavera G, Castresana J. Improvement of phylogenies after removing divergent and ambiguously aligned blocks from protein sequence alignments. Syst Biol. 2007;56:564-77.

24. Stamatakis A. RAxML version 8: a tool for phylogenetic analysis and postanalysis of large phylogenies. Bioinformatics. 2014;30:1312-3.

25. Huson D, Linz S. Autumn Algorithm - Computation of Hybridization Networks for Realistic Phylogenetic Trees. IEEE/ACM Trans Comput Biol Bioinform. 2016. doi:10.1109/TCBB.2016.2537326

26. Aziz RK, Bartels D, Best AA, DeJongh M, Disz T, Edwards RA, et al. The RAST Server: rapid annotations using subsystems technology. BMC Genomics. 2008:9:75.

27. Chen Y, Chatterjee SS, Porcella SF, Yu YS, Otto M. Complete genome sequence of a Panton-Valentine leukocidin-negative community-associated methicillin-resistant Staphylococcus aureus strain of sequence type 72 from Korea. PLoS One. 2013;8:e72803.

28. Diep BA, Gill SR, Chang RF, Phan TH, Chen JH, Davidson MG, et al. Complete genome sequence of USA300, an epidemic clone of community-acquired meticillin-resistant Staphylococcus aureus. Lancet. 2006;367:731-9.

29. Machuca MA, Sosa LM, Gonzalez Cl. Molecular typing and virulence characteristic of methicillin-resistant Staphylococcus aureus isolates from pediatric patients in Bucaramanga, Colombia. PLoS One. 2013;8:e73434.

30. Portillo BC, Moreno JE, Yomayusa N, Alvarez CA, Cardozo BE, Perez JA, et al. Molecular epidemiology and characterization of virulence genes of community-acquired and hospital-acquired methicillin-resistant Staphylococcus aureus isolates in Colombia. Int J Infect Dis. 2013;17:e744-9. 
31. Planet PJ, Diaz L, Kolokotronis SO, Narechania A, Reyes J, Xing G, et al. Parallel Epidemics of Community-Associated Methicillin-Resistant Staphylococcus aureus USA300 Infection in North and South America. J Infect Dis. 2015;212:1874-82.

32. Rodriguez EA, Correa MM, Ospina S, Atehortua SL, Jimenez JN. Differences in epidemiological and molecular characteristics of nasal colonization with Staphylococcus aureus (MSSA-MRSA) in children from a university hospital and day care centers. PLoS One. 2014;9:e101417.

33. Deghorain M, Van Melderen L. The Staphylococci phages family: an overview. Viruses. 2012;4:3316-35.

34. Arias CA, Rincon S, Chowdhury S, Martinez E, Coronell W, Reyes J, et al. MRSA USA300 clone and VREF-a U.S.-Colombian connection? N Engl J Med. 2008;359:2177-9.

35. Coombs GW, Monecke S, Pearson JC, Tan HL, Chew YK, Wilson L, et al. Evolution and diversity of community-associated methicillin-resistant Staphylococcus aureus in a geographical region. BMC Microbiol. 2011;11:215.

Submit your next manuscript to BioMed Central and we will help you at every step:

- We accept pre-submission inquiries

- Our selector tool helps you to find the most relevant journal

- We provide round the clock customer support

- Convenient online submission

- Thorough peer review

- Inclusion in PubMed and all major indexing services

- Maximum visibility for your research

Submit your manuscript at www.biomedcentral.com/submit
Biomed Central 